\title{
Informationswissenschaft als Hochschulstudium - bereit für die Zukunft in Gesellschaft, Industrie und Wissenschaft
}

DOI 10.1515/iwp-2015-0037

Die Fachrichtung Informationswissenschaft ist mit wenigen Worten nicht zu erklären. Diese vermeintliche Schwäche ist im Grunde jedoch eine Stärke: Die Studierenden bekommen eine solide Grundlagenausbildung mit technologischem Fokus und sind vielfältig einsetzbar. Das Fach selbst kann und muss regelmäßig seine Inhalte überarbeiten und an die Erfordernisse aus Gesellschaft und Arbeitswelt anpassen. Neben den Grundlagen stehen innovative forschungsorientierte und projektbasierte Lehrinhalte.

Deskriptoren: Informationswissenschaft, Hochschulausbildung, Lehrplan, Semantik, Beruf

Information science as university education - prepared for the future in society, industry and academic environments

Information Science can be complicated to explain. This obvious weakness is in fact an asset of the science: Students get a solid basic education with a technological focus and are versatile. Information Science can and must review its content and adapt to the requirements of society and the working environment on a regular basis. Besides the solid basics, the curriculum contains innovative knowledge-driven and project-based content.

Descriptors: Information science, University education, Curriculum, Semantics, Occupation

Les sciences de l'information en tant qu'études universitaires - prêtes pour l'avenir dans la société, l'industrie et les sciences

La branche des sciences de l'information ne peut être expliquée en quelques mots. Cette faiblesse prétendue est en fait une force: Les étudiants obtiennent une éducation de base solide avec un accent technologique et deviennent dès lors polyvalents. La matière, quant à elle, peut et doit être remaniée régulièrement au niveau de son contenu et s'adapter aux exigences de la société et du monde du travail. À côté des bases, on y trouve des contenus de cours innovateurs orientés vers la recherche et basés sur des projets.

Descripteurs: sciences de l'information, enseignement supérieur, programmes d'études, exigence

\section{Einleitung}

Wenn man Studierende der Informationswissenschaft fragt, was sie eigentlich studieren, bekommt man selten eine umfassende, klare und einheitliche Antwort. Es steht zu befürchten, dass das Ergebnis bei einer Befragung der Lehrenden nur wenig besser aussieht.

Das Fach Informationswissenschaft gibt es an Universitäten und Fachhochschulen bereits seit vielen Jahren. Einige Hochschulen (wie z. B. die Universität Saarbrücken) sahen keine Zukunft in diesem Fach und haben den Studiengang geschlossen. Andere Hochschulen (wie z. B. die Hochschule Darmstadt) haben beschlossen, die Inhalte des Studiengangs immer wieder zu reformieren und für die Anforderungen in Gesellschaft, Industrie und Wissenschaft fit zu machen.

Eine Absolventenbefragung aus dem Frühjahr 2014 hat gezeigt, dass das Studium die Absolventen der Hochschule Darmstadt effektiv auf Führungspositionen, nicht nur in Bibliotheken, sondern zunehmend auch in der datenverarbeitenden Industrie vorbereitet. Beim Darmstädter Symposium Informationswissenschaft haben die Studierenden eine Vielfalt ihrer Projekte gezeigt, die bei den anwesenden Firmen aus dem IT-Bereich auf reges Interesse gestoßen ist. Die Vielfalt und die Interdisziplinarität des Faches sollten daher als Chance gesehen werden, 
und nicht etwa als Manko. Die immer wieder neu notwendige Definition des Faches mit einem Bezug auf gesellschaftliche Entwicklungen ist eine große Stärke der Informationswissenschaft.

Dieser Artikel gibt einige Beispiele dafür, was die Informationswissenschaft im Moment für Gesellschaft und Arbeitswelt beiträgt, und Hinweise darauf, wie die Lehre (und damit auch das Fachgebiet) weiterentwickelt werden kann.

Dafür beschäftigen wir uns zunächst mit den Kernthemen der Informationswissenschaft, analysieren dann, wie die Lehre der Informationswissenschaft zum jetzigen Zeitpunkt für Gesellschaft und Arbeitsmarkt vorbereitet und zeigen schließlich einen Weg, wie das Studienfach auch für die Zukunft gerüstet sein kann.

\section{Information}

Die Informationswissenschaft arbeitet - wie auch der Name schon sagt - im Kern mit Information. Dabei geht es zunächst um die Strukturierung und Darstellung von Information in Datenbanken, Thesauren und Ontologien, aber auch um die Visualisierung. Automatische Verfahren zum Aufbau und zur Nutzung dieser strukturierten Darstellungen stehen im Vordergrund.

Hinzu kommt die Suche nach Information, klassisch im Information Broking, aber verstärkt im Information Retrieval, das auf automatische Verfahren fokussiert ist. Automatische Verfahren der Informationsextraktion gehen heute über die Suche nach Stichwörtern hinaus und binden ontologische (semantische) Information ein, extrahieren semantische Relationen aus Textdaten und erzeugen automatische Textzusammenfassungen, um nur einige Beispiele zu nennen.

Der dritte Bereich ist die Erzeugung von Information. Hier geht es zunächst darum, qualitativ hochwertige Information $\mathrm{zu}$ erzeugen, also Informationsqualität sicherzustellen (z. B. tekom (2013)). Dazu kommen die Strukturierung von Information mit Bezug auf ihre Funktion (z. B. Muthig und Schäflein-Armbruster 2008) und auch die Ideen der semantischen Annotation (Berners-Lee et al. 2001). Schließlich gehört in diesen Bereich auch die Übersetzung von Information, deren Unterstützung durch automatische Verfahren längst Standard geworden ist.

In sehr vielen dieser Fälle handelt es sich um Information, die in sprachlicher Form verfügbar ist (Suche oder Strukturierung) oder die als sprachliche Information erzeugt wird.

\section{Sprache und Semantik}

Ein großer Anteil der Information, mit der wir uns beschäftigen, ist in sprachlicher Form kodiert, sehr oft als unstrukturierter Text ${ }^{1}$. Die Information der sprachlichen Zeichen (Buchstaben) im Text kann erst dadurch gewonnen werden, dass die Bedeutung der Sprache - also ihre Semantik - bestimmt wird. Das, was wir Menschen intuitiv tun, ist für eine Maschine eine erhebliche Rechenleistung. Wie soll z. B. eine Maschine automatisch erkennen, was in einer "Mädchenhandelsschule“ gelehrt wird? Dazu gehört kulturelles Wissen und Wissen über den Kontext, in dem das Wort auftritt.

Information ist aber auch erst dann wirklich wertvoll, wenn sie vernetzt wird. Ein Beispiel: Ein Immobilienmakler hat Informationen über Wohnungen und Häuser, die er in seiner Datenbank hat: Die Lage, die Anzahl der Zimmer, das Vorhandensein von Garten oder Balkon, Garagen usw. Er hat auch Informationen über die potentiellen Mieter und Käufer: Die Anzahl der Personen, die finanziellen Möglichkeiten, Wünsche an die neue Wohnung usw. Erst wenn er diese Informationen miteinander verbindet, kann er einen Mehrwert daraus ziehen, indem er z. B. weiß, dass ein Mieter Kinder hat und daher die Lage seiner neuen Wohnung in einer ruhigen Wohnstraße bevorzugt.

Diese inhaltliche Vernetzung von Information ist Semantik. Eine Grundlagenveranstaltung im ersten und zweiten Semester Informationswissenschaft beschäftigt sich daher mit den zentralen Fragen: Was ist Bedeutung? Was kann man mit der semantischen Analyse machen²?

\section{Texttechnologie}

Wir Menschen können Sprache intuitiv verstehen und verarbeiten. Wir können - wie der Immobilienmakler in unserem Beispiel - Information aus sprachlichen Zeichen extrahieren und miteinander vernetzen. Dabei sind wir in der Lage, erhebliche semantische Inferenzen zu ziehen. Im Zeitalter großer Datenmengen, die durchsucht, strukturiert, verstanden und auch produziert werden müssen, sind wir aber auf Maschinen angewiesen, die uns dabei

1 Selbstverständlich sind auch strukturierte Information (z. B. in Datenbanken) und Information in Form von Bild oder Ton Gegenstand der Informationswissenschaft, werden in diesem Artikel aber zunächst nicht betrachtet.

2 NB: Es gibt nicht nur eine Sprache, d. h. wir müssen uns auch mit Mehrsprachigkeit und Sprachvergleich befassen. 
unterstützen. So ist es für einen Redakteur in einer großen Fernsehanstalt nicht möglich, täglich alle (möglichst weltweiten) Pressemitteilungen zu seinem Themengebiet $\mathrm{zu}$ lesen. Für diese Aufgabe gibt es die DokumentarAbteilung. Aber auch dort würde man der Menge der Informationen, die täglich anwächst, nicht gerecht, würde man nicht automatische Verfahren zur Indexierung, zur Suche und zur Vernetzung von Information einsetzen.

Für die Informationswissenschaft bedeutet das, einen Schwerpunkt auf Technologie zu setzen, die die Bedeutung (die Semantik) der Sprache automatisch verarbeiten kann - um Information aus Sprache zu generieren: Texttechnologie.

Bei der Texttechnologie handelt es sich zunächst um Sprachtechnologie, wie z. B. Technologie zur Verarbeitung von Morphologie, automatische Erkennung von Namen, Erkennung von Kollokationen (Wortpaaren), Erkennung von Wortvarianten, automatische Auflösung von Mehrdeutigkeiten, semantischer Analyse von Sätzen, Lernen von semantischen Zusammenhängen aus annotierten Texten und vieles mehr. Wir brauchen auch Technologie, um Texte und Informationen zu strukturieren und um die Bedeutung darzustellen, wie z. B. XML, Meta-Tags und Ontologie-Beschreibungssprachen.

\section{Industrielle Anwendungen der Gegenwart}

Nun lehren und forschen wir in und als Teil einer Gesellschaft. Gerade als Hochschule für angewandte Wissenschaft und als Vertreter einer angewandten Wissenschaft setzen wir uns damit auseinander, was unsere Studierenden auf dem Arbeitsmarkt brauchen und was die Informationswissenschaft für die Gesellschaft beitragen kann.

Der erste Kontakt der Studierenden mit dem Arbeitsmarkt ist das Praktikum. Eine Analyse der Praktikumsberichte der letzten zwei Jahre zeigt, in welchen Berufsfeldern Informationswissenschaftler eingesetzt werden können und welche Fertigkeiten dafür benötigt werden.

Zunächst fällt auf, dass der Bereich, in dem Informationswissenschaftler mit einer stark technischen Ausrichtung eingesetzt werden können, sehr breit ist. Das ist einerseits der Grund dafür, dass es notwendig ist, das Fachgebiet immer wieder neu zu erklären, aber auch neu zu definieren. Andererseits ist es eine Stärke des Fachgebiets, dass die Absolventen breit einsetzbar sind.

Es zieht sich durch die Praktikumsberichte, dass gerade die Inhalte der technischen Fächer in der Praxis gebraucht werden. Die Studierenden bemerken das oft erst hier durch den Praxisschock. Ein Student ${ }^{3}$ schreibt: „Da meine Kenntnisse in der Programmierung leider nicht besonders ausgeprägt waren, war es mir nicht möglich mich mehr in das Projekt einzubinden. Für mich persönlich habe ich dann an dieser Stelle ein Defizit festgestellt, welches ich in der Zukunft anzugehen gedenke.“

Innovative Themen finden sich zum Teil in der Rubrik „zusätzliche Inhalte“, wie „Internet der Dinge“, „Industrie 4.0“ und „semantische Datenmodelle“.

Es finden sich aber auch Rückmeldungen wie „Keine Aufgabe war mir völlig neu und es war gut, zu sehen, dass das Studium mich auf viele Aufgabenfelder im Berufsleben gut vorbereitet hat.“

Es zeigt sich, dass es lohnenswert ist, diese Rückmeldungen aus der Praxis gründlich zu lesen.

Wir sehen klar: Für industrielle Anwendungen der Gegenwart brauchen unsere Studierenden solide technische Grundkenntnisse. Daher sollten wir die technologischen Anteile mit einem Schwerpunkt auf die Texttechnologie in der Informationswissenschaft noch mehr stärken als wir das schon tun. Diese Stärkung kann in einer besseren Vernetzung der Studieninhalte bestehen, wobei die Voraussetzung dafür die intensivere Kommunikation der Lehrenden ist.

Unsere Studieninhalte - so zeigen die Praktikumsberichte - sind für die industriellen Anwendungen der Gegenwart nützlich und wir arbeiten in die richtige Richtung. Auch wenn viele unserer Studierenden die technischen Fächer nicht sehr mögen, sehen sie im Praktikum, dass diese Kenntnisse nützlich sind. Dieser Kontakt mit der Praxis (der „Praxisschock“) kommt aber für die Studierenden zu spät - in der Abschlussphase ihres Studiums. Der Kontakt zur Praxis sollte durch regelmäßige Gespräche mit und Besuchen in Firmen frühzeitig hergestellt werden. Ein erster Schritt in diese Richtung ist die beginnende Kooperation zwischen dem Studiengang Informationswissenschaft und dem Verein IT-for-Work.

\section{Industrielle Anwendungen der Zukunft}

Durch Praktikumsberichte und Absolventenbefragungen bekommen wir ein recht klares Bild, was die Studierenden im Moment brauchen, um in der Arbeitswelt beste-

3 Die Studierenden, die hier zitiert werden, bleiben anonym. In diesem Fall handelt es sich um einen Studenten im Masterstudium. der die letzte Änderung der Studienstruktur im Bachelorstudium mit einem stärkeren Fokus auf moderne Technologie nicht erlebt hat. 
Tabelle 1: Analyse von Praktikumsberichten der Hochschule Darmstadt zu Berufsfeldern und benötigten Fertigkeiten 2013 bis 2015.

\begin{tabular}{|c|c|c|}
\hline Berufsfeld & Benötigte Studieninhalte & $\begin{array}{l}\text { Zusätzliche Inhalte, die benötigt wurden, } \\
\text { aber nicht gelehrt wurden }\end{array}$ \\
\hline $\begin{array}{l}\text { Webseitenentwicklung, } \\
\text { Online-Agenturen }\end{array}$ & $\begin{array}{l}\text { PHP } \\
\text { CSS } \\
\text { HTML } \\
\text { JavaScript } \\
\text { Webscripting } \\
\text { Datenbanken } \\
\text { Content Management System } \\
\text { Informatik } \\
\text { Projektmanagement }\end{array}$ & $\begin{array}{l}\text { Bildbearbeitung } \\
\text { neue Programmiersprachen } \\
\text { mehr Content Management Systeme }\end{array}$ \\
\hline Suchmaschinenoptimierung & $\begin{array}{l}\text { Statistik } \\
\text { Information Retrieval } \\
\text { Informationsdesign } \\
\text { Suchmaschinenoptimierung } \\
\text { Content Management System } \\
\text { Informatik } \\
\text { HTML } \\
\text { Datenbanken } \\
\text { Medieninformation }\end{array}$ & mehr Informatik \\
\hline Information in Bibliotheken und Verlagen & $\begin{array}{l}\text { Datenbanken } \\
\text { Content Management } \\
\text { PHP } \\
\text { XML, XSLT und Metadaten } \\
\text { Wissensrepräsentation } \\
\text { Inhaltserschließung }\end{array}$ & $\begin{array}{l}\text { weitere Programmiersprachen } \\
\text { Bibliotheksgeschichte } \\
\text { Öffentliches Archivwesen } \\
\text { Bildbearbeitung }\end{array}$ \\
\hline Herstellen von Informationsprodukten & $\begin{array}{l}\text { Sprachkontrolle } \\
\text { Informationsqualität }\end{array}$ & \\
\hline Wissensmanagement & $\begin{array}{l}\text { Modellierung von Begriffssystemen } \\
\text { semantische Netze } \\
\text { Linked Data } \\
\text { Information Retrieval } \\
\text { Semantik } \\
\text { Ontologien } \\
\text { Programmierung } \\
\text { Datenbanken } \\
\text { Metadaten }\end{array}$ & $\begin{array}{l}\text { Semantische Datenmodelle } \\
\text { Logik von Begriffshierarchien und Ontologien } \\
\text { persönliches Zeitmanagement } \\
\text { Projektdokumentation } \\
\text { Datenbankentwicklung }\end{array}$ \\
\hline Projektorganisation & $\begin{array}{l}\text { Projektmanagement } \\
\text { Projektdokumentation } \\
\text { Projektplanung } \\
\text { Zeitmanagement }\end{array}$ & \\
\hline $\begin{array}{l}\text { Informationsrecherche und } \\
\text { Business Intelligence }\end{array}$ & $\begin{array}{l}\text { Information Broking } \\
\text { VWL und BWL } \\
\text { Relationale Datenbanken } \\
\text { Informationsdesign } \\
\text { Statistik } \\
\text { Wirtschafts- und Finanzinformation } \\
\text { Business Intelligence }\end{array}$ & $\begin{array}{l}\text { Business Intelligence Software } \\
\text { mathematische Hintergründe } \\
\text { Möglichkeiten der Datenmodellierung } \\
\text { Industrie 4.0; Internet der Dinge }\end{array}$ \\
\hline
\end{tabular}

hen zu können. Wir wollen aber nicht nur Absolventen ausbilden, die jetzt und in den nächsten fünf Jahren in ihrem Job bestehen können, sondern wir wollen die Studierenden so ausbilden, dass sie auch in zehn oder zwanzig Jahren als Informationswissenschaftler arbeiten können und dann in der Lage sind, sich und das Fach weiter $\mathrm{zu}$ entwickeln. Das heißt, wir wollen einerseits Menschen ausbilden, die auf der einen Seite neue Trends 
Tabelle 2: Drei Säulen innovativer und forschungsorientierter informationswissenschaftlicher Lehre.

\begin{tabular}{lll}
\hline $\begin{array}{l}\text { Informationswissenschaftliches } \\
\text { Kolloquium }\end{array}$ & Forschungskolloquium & $\begin{array}{l}\text { Forschungsprojektorientierte } \\
\text { Lehre }\end{array}$ \\
\hline$-\quad$ Rezeption und Bewertung & $-\quad$ Entwickeln von Visionen & - Vorantreiben von Forschungsprojekten \\
$\quad$ von Forschungsarbeiten & $-\quad$ Erarbeitung und ständiges Update einer & - Ausarbeitung neuer Forschungsideen \\
& & Roadmap für die Informationswissenschaft \\
\hline
\end{tabular}

erkennen, die sich in Dinge neu einarbeiten können. Andererseits wollen wir Menschen ausbilden, die selbst Visionen entwickeln können und die selbst die Informationswissenschaft mitgestalten können.

Das führt uns zum Bereich Wissenschaft. Da geht es um nichts anderes als darum, Visionen zu haben und auszubauen. Die Dinge, die in der Wissenschaft entwickelt werden oder auch ausprobiert werden, sind nicht Dinge, die nächstes Jahr direkt in der Industrie eingesetztwerden. Ein Beispiel aus der Wissenschaftsgeschichte: In den 1990er Jahren gab es in Deutschland ein großes Projekt zur Maschinellen Übersetzung, Verbmobil. ${ }^{4}$ Das war damals weit entfernt von industrieller Anwendung, ein visionäres Projekt. Heute ist die Maschinelle Übersetzung zum größten Teil ein gelöstes Problem. Heute unterrichtet man die Techniken unter dem Aspekt von Techniken, die in der Industrie eingesetzt werden und die man deshalb eben kennen muss.

Genau so müssen wir auch vorgehen. Wir erarbeiten Visionen. Das Schöne an der Arbeit in der Hochschule ist, dass wir mit jungen Leuten arbeiten. Junge Leute sind besonders dazu in der Lage, Visionen zu entwickeln. Wir geben ihnen die notwendige Hintergrundinformation und versetzen sie in die Lage, weiter zu denken: Wohin wollen wir? Was könnte man damit eigentlich auch noch machen? Wo könnte das hingehen?

Notwendig ist es dabei, weiter zu denken, ohne ständig eine Schere im Kopf zu haben: Das geht sowieso nicht. Es gab einen Report in den 1960ern ${ }^{5}$, in dem gesagt wurde, dass die Maschinelle Übersetzung nie funktionieren wird, weil Sprache zu komplex ist. Trotzdem haben die Wissenschaftler in den 1990ern in Deutschland wieder angefangen $\mathrm{zu}$ forschen und sind sehr weit gekommen. Das bedeutet, wir dürfen den Bereich Forschung und Wissenschaft nicht vernachlässigen. Wir müssen den Studierenden beibringen, neugierig zu sein. Wir müssen ihnen zeigen, dass wir Visionen haben und sie versuchen umzusetzen. Und wir müssen ihnen dann auch beibringen, selbst visionär zu denken.
Mein Vorschlag dazu ist: Das Etablieren von mehreren weiteren kleinen Forschungsprojekten, die wir von der Informationswissenschaft aus tragen. Das muss nicht unbedingt immer geförderte Forschung sein. Wir können auch Forschungsprojekte erst mal aufsetzen und dann zu einem späteren Zeitpunkt entscheiden, ob wir eine Förderung dafür beantragen. Wir treiben diese Projekte mit Studierenden voran und schauen dann an einem bestimmten Punkt, ob wir weiter machen und öffentliche Fördermittel einwerben.

Einige Beispiele für solche Forschungsprojekte sind:

- Leichte Sprache und deren Unterstützung durch Technologie $^{6}$

- Informationsextraktion aus Geschäftsberichten

- Opinion Mining für die deutsche Sprache

- Neue Methoden der Visualisierung von Wissen

- Neue Methoden der Archivierung von und des Zugangs zu Daten

- Ontologie-Aufbau aus Sprachdaten wie z. B. Wikipedia

- Trenderkennung aus Social Media-Daten

Innovative und forschungsorientierte Lehre sollte aus drei Säulen bestehen (s. Tabelle 2).

\section{Fazit}

Die Informationswissenschaft ist eine Fachrichtung, die sich - vor allem aufgrund ihrer Interdisziplinarität nicht mit wenigen Worten erklären lässt. Diese vermeintliche Schwäche der Fachrichtung ist jedoch ihre Stärke: Die Studierenden bekommen eine breite Grundlagenausbildung mit technologischem Fokus und sind vielfältig einsetzbar. Das Fach selbst kann und muss regelmäßig seine Inhalte überarbeiten und an die Erfordernisse aus Gesellschaft und Arbeitswelt anpassen. Dadurch (und auch durch einen starken Schwerpunkt auf innovative Technologie) entsteht ein Zwang zur Innovation und zur ständigen Diskussion. Visionäres wissenschaftliches Ar-

6 Siegel und Lieske (2014)
4 Wahlster (2000)

5 ALPAC (1966) 
beiten ist notwendig, um das Fach voranzutreiben, und damit auch die Studierenden auch in Zukunft bestehen zu können.

\section{Literatur}

ALPAC (1966): Language and Machines. Computers in Translation and Linguistics, A Report by the Automatic Language Processing Advisory Committee (ALPAC), National Academy of Sciences - National Council, Washington, D. C.

Berners-Lee, T., Hendler, J., \& Lassila, O. (2001): The Semantic Web. Scientific American, 284(5), 28-37.

Muthig, J., \& Schäflein-Armbruster, R. (2008): Funktionsdesign ${ }^{\circledR}$ methodische Entwicklung von Standards. Standardisierungsmethoden für die Technische Dokumentation, tekom-Hochschulschriften, 16.

Siegel, Melanie und Lieske, Christian (2014): Beitrag der Sprachtechnologie zur Barrierefreiheit: Unterstützung für Leichte Sprache. Symposium 11: Barrierefreie Sprache in der digitalen Kommunikation für Öffentlichkeit, Institutionen und Unternehmen auf der GAL-Tagung 2014 in Marburg. tekom (Hg.) (2013): Leitlinie. Regelbasiertes Schreiben. Deutsch für die Technische Kommunikation. Stuttgart. 2. erweiterte Auflage.

Wahlster, W. (ed.) (2000): Verbmobil: Foundations of Speech-toSpeech Translation. Berlin: Springer Verlag.

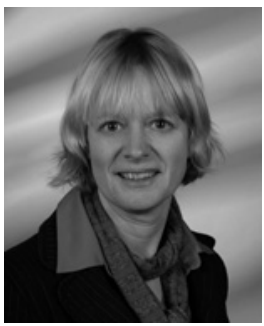

Prof. Dr. Melanie Siegel

Hochschule Darmstadt

Campus Dieburg

Max-Planck-Straße 2

64807 Dieburg

melanie.siegel@h-da.de

www.melaniesiegel.de

Dr. Melanie Siegel ist Professorin für Informationswissenschaft an der Hochschule Darmstadt. Sie interessiert sich für Sprachtechnologie, Maschinelle Übersetzung, Syntax und Semantik der japanischen Sprache, Ontologien, Informationsextraktion, Sentimentanalyse und Technische Dokumentation. Ihre Stationen: Studium, Promotion und Habilitation an der Universität Bielefeld, Forschungsarbeiten an der Universität Saarbrücken und dem Deutschen Forschungszentrum für Künstliche Intelligenz, Forschung und Entwicklung bei der Berliner Firma Acrolinx GmbH, mehrmonatige Auslandsaufenthalte in den USA und in Japan. 